\title{
Efeito isolado e combinado do sexo, tipo de esporte e experiência competitiva sobre as habilidades psicologicas
}

Unique and combined effect of gender, type of sport and competitive experience on psychological skills

\author{
Marcos Gimenes Fernandes \\ Universidade Estadual de Santa Cruz, Brasil \\ gimenes@uesc.br
}

Izamar dos Santos Abreu

Universidade Estadual de Santa Cruz, Brasil

\author{
Jehnnifer Alcantara \\ Universidade Estadual de Santa Cruz, Brasil \\ Sandra Adriana Neves Nunes \\ Universidade Federal do Sul da Babia, Brasil
}

\section{Resumo:}

As habilidades psicológicas podem ter efeitos de variáveis nominais isoladas e/ou combinadas explicando de alguma forma a sua variabilidade. O presente estudo teve os seguintes objetivos: i) examinar os efeitos isolados do contexto, experiência competitiva, tipo de esporte e do sexo sobre o uso de habilidades psicológicas, ii) verificar o efeito combinado ou interativo entre sexo, experiência competitiva e tipo de esporte no uso das habilidades psicológicas e iii) investigar o poder preditivo das variáveis explicativas (sexo, tipo de esporte e experiência competitiva) sobre as variáveis psicológicas, nos contextos de treinamento e de competição. Os participantes foram 357 atletas entre 18 e 40 anos $(M=25,69$, DP =5,61), sendo 278 do sexo masculino e 79 do sexo feminino. A versão brasileira do Test of Performance Strategies-2 foi aplicado uma hora antes da competição. O sexo, tipo de esportes e experiência competitiva tiveram efeito significativo no relaxamento, controle emocional e definição de objetivos. Sexo também foi o único preditor de relaxamento, na situação de treino, e de controle emocional e de relaxamento, na situação de competição. Tipo de esporte e experiência competitiva foram bons preditores de definição de objetivos, na situação de treino, enquanto que na situação de competição, apenas experiência competitiva predisse definição de objetivos. Além disso, o contexto (treino ou competição) teve efeito significativo sobre definição de objetivos, automatização, relaxamento e imagética. A interação entre sexo/tipo de esportes teve efeito sobre diálogo interno (treino), e tipo de esportes/experiência competitiva teve efeito sobre o relaxamento (treino e competição) e ativação (competição). Finalmente, experiência competitiva/sexo teve efeito significativo e combinado sobre definição de objetivo (treino). Esses resultados foram discutidos, tendo em conta suas implicações teóricas e práticas para o planejamento de intervenções da Psicologia do Esporte no Brasil.

Palavras-chave: TOPS-2, Habilidades psicológicas, Sexo, Experiência competitiva, Tipo de esporte.

\section{Abstract:}

Psychological skills may have effects of isolated and/or combined nominal variables explaining their variability in some way. The present study had the following objectives: i) to examine the unique effect of context, competitive experience, type of sport and gender on the use of psychological abilities, ii) to verify the combined or interactive effect of gender, competitive experience and type of sport on psychological skills and iii) to investigate the predictive power of the explanatory variables (gender, type of sport and competitive experience) on the psychological variables, in the contexts of training and competition. The participants were 357 athletes between 18 and 40 years old $(M=25.69, S D=5.61), 278$ males and 79 females. The Brazilian version of the Test of Performance Strategies-2 was applied one hour before the competition. Gender, type of sports and competitive experience had significant effect on relaxation, emotional control and goal setting. Gender was also the only predictor of relaxation, in the training situation, and of emotional control and of relaxation, in the competitive situation. Type of sport and competitive experience were good predictors of goal setting in the training situation, while in the competitive situation, only competitive experience predicted goal setting. In addition, the context (training or competition) had a significant effect on goal setting, automaticity, relaxation and imagery. The interaction between gender/type of sports had an effect on self talk (training), and type of sports/competitive experience had an effect on relaxation (training and competition) and activation (competition). Finally, competitive experience/ gender had a significant and combined effect on goal setting (training). These results were discussed, taking into account the theoretical and practical implications for the planning of interventions in the field of Sports Psychology in Brazil.

KEYwORDs: TOPS-2, Psychological skills, Gender, Competitive experience, Type of sport.

\section{Recepción: 30 de julio de 2018 | Aprobación: 03 de febrero de 2019| Publicación: 05 de abril de 2019}

Cita sugerida: Gimenes Fernandes, M., dos Santos Abreu, I y Alcantara, J. (2019). Efeito isolado e combinado do sexo, tipo de esporte e experiência competitiva sobre as habilidades psicológicas. Educación Física y Ciencia, 21(2), e079. https://doi.org/10.24215/23142561e079 


\section{INTRODUÇÃO}

De acordo com Gould e Weingberg (2015), habilidades psicológicas no contexto esportivo são competências que podem ser desenvolvidas e treinadas como as habilidades físicas. Gould, Flett e Bean (2009) afirmam que os técnicos reconhecem a importância da preparação psicológica para atletas, a qual está relacionada ao termo treinamento de habilidades psicológicas (THP). Para Vealey (2007) o THP tem como objetivo auxiliar os atletas a melhorarem o seu desempenho através do uso de técnicas psicológicas para aumentarem as suas habilidades psicológicas. Especificamente, técnicas psicológicas como definição de objetivos, imagética e relaxamento são utilizadas para desenvolver habilidades psicológicas como concentração e autoconfiança (Burton, \& Raedeke, 2008). De acordo com Craft, Magyar, Becker e Feltz (2003), a autoconfiança é um determinante do desempenho esportivo. No contexto competitivo, Fernandes, Nunes, Vasconcelos-Raposo, Fernandes e Brustad (2013) e Fernandes, Vasconcelos-Raposo e Fernandes (2012) relatam que a autoconfiança ajuda os atletas a controlarem e diminuírem seus níveis de ansiedade cognitiva e somática. Dessa forma, evidências empíricas revelam que o treinamento de habilidades psicológicas pode trazer inúmeras vantagens para os atletas de competição.

Diversos estudos tiveram como objetivo investigar o THP no contexto esportivo. Por exemplo, Weinberg Stitcher, \& Richardson (1994) investigou como a definição de objetivos afeta o desempenho do esporte e Neil, Mellalieu e Hanton (2006) estudaram as relações entre habilidades psicológicas e ansiedade competitiva. Além disso, Horn, Gilbert, Gilbert e Lewis (2011) estudaram as habilidades psicológicas em atletas colegiais, enquanto Freitas, Dias e Fonseca (2013) estudou o uso de habilidades psicológicas por atletas de elite. Mais recentemente, Hagan, Pollmann e Schack (2017) investigaram a implementação e sistematização do desenvolvimento de habilidades psicológicas em um ciclo contínuo de treinamento, Kristjansdottir, Erlingsdottir, Sveinsson e Saavedrav (2018) analisaram as habilidades psicológicas em função da idade e do sexo em atletas de elite e Wilson, Bennett, Mosewich, Faulkner e Crocker (2019) que exploraram a percepção da experiência em habilidades psicológicas no sucesso competitivo e no controle do estresse.

No que diz respeito às habilidades psicológicas mais estudadas na literatura, a definição de objetivos, o relaxamento e o diálogo interno têm recebido destaque por serem importantes durante a competição e melhorarem o desempenho competitivo (Sadeghi, Omar-Fauzee, Jamalis, Ab-Latif, \& Cheric, 2010). De acordo com Burton e Raedeke (2008), definição de objetivos tem efeito sobre o desempenho direcionando a atenção, mobilizando esforços, aumentando a persistência e desenvolvendo estratégias de motivação. Por outro lado, a utilização de técnicas de relaxamento melhora o desempenho esportivo (Newmark, \& Bogacki, 2005), reduz a ansiedade e aumenta a autoconfiança (Kulmatycki, \& Bukowska, 2007). Por fim, dialogo interno é definido como verbalizações endereçadas a si próprio e serve de funções instrucionais e motivacionais para atletas (Edwards, Tod, \& McGuigan, 2008), e aumenta o desempenho e habilidades no esporte (Hardy, 2006).

Com o objetivo de instrumentalizar a avaliação de diversas habilidades psicológicas relacionadas ao desempenho competitivo de atletas, Hardy, Roberts, Thomas e Murphy (2010) desenvolveram o Test of Performance Strategies-2 (TOPS-2). Esse instrumento verifica diversas habilidades psicológicas em situação de treino e de competição. No Brasil, Fernandes, Vasconcelos-Raposo e Fernandes (2013) recorreram a procedimentos de Análise Fatorial Confirmatória (AFC) para verificar as propriedades psicométricas da versão brasileira do TOPS-2. Os resultados revelaram bons índices de confiabilidade $(\alpha>0,70)$, bem como, satisfatórios índices de ajustamento para a situação de treino $(\mathrm{GFI}=0,953$; $\mathrm{CFI}=0,944$; $\mathrm{RMSEA}=0,046)$ e para a situação de competição $(\mathrm{GFI}=0,941$; $\mathrm{CFI}=0,945$; $\mathrm{RMSEA}=0,045)$. 
Thomas, Murphy e Hardy (1999) tiveram como um dos objetivos de estudo, verificar a correlação entre as habilidades psicológicas nas situações de treino e competição. Os resultados revelaram correlações positivas significativas entre diálogo interno e ativação, imagética, definição de objetivos, relaxamento e controle emocional para a situação de competição, por outro lado, diálogo interno foi correlacionado negativamente com pensamentos negativos e automaticidade. Para a situação de treino, os resultados revelaram resultados semelhantes à situação de competição, como por exemplo, diálogo interno foi positivamente correlacionado com relaxamento, imagética, definição de objetivos, ativação, controle atencional e controle emocional. Quando os autores tiveram como objetivo correlacionar às habilidades psicológicas entre treino e competição, os resultados demonstraram correlações significativas para dialogo interno, imagética, definição de objetivos, ativação, automaticidade e relaxamento. Pensamento negativo foi correlacionado negativamente para controle emocional e controle atencional, na situação de treino.

No que diz respeito ao efeito da situação de treino e da competição na utilização das habilidades psicológicas, Lane, Harwood, Terry e Karageorghis (2004) e Fernandes, Vasconcelos-Raposo e Fernandes (2013) relataram em seus resultados que houve diferença significativa na utilização das habilidades psicológicas nas situações de treino e competição. Com relação ao efeito da experiência competitiva, Neil, Mellalieu e Hanton (2006) reportaram que os atletas de elite revelaram maior uso de relaxamento, imagética e diálogo interno do que os atletas menos experientes.

Vale destacar que até a presente data os estudos dessa área verificaram o efeito isolado do sexo, experiência competitiva e tipo de esporte sobre as habilidades psicológicas. Estudos recentes (Fernandes et al., 2013; Hagan et al., 2017) sugerem que é necessária uma análise dos efeitos combinados ou interativos dessas variáveis psicológicas sobre o desempenho esportivo. No Brasil há uma carência de estudos que tenham tido como objetivo avaliar o efeito da interação dessas variáveis independentes sobre as dimensões do TOPS-2. Dessa forma, o presente estudo teve os seguintes objetivos, a saber: i) examinar o efeito do contexto (treino $v s$ competição), experiência competitiva (baixa $v s$ alta), tipo de esporte (individual $v s$ coletivo) e do sexo (masculino $v$ feminino) no uso das habilidades psicológicas, ii) verificar o efeito combinado ou interativo das variáveis sexo, experiência competitiva e tipo de esporte no uso das habilidades psicológicas e iii) investigar o poder preditivo das variáveis explicativas (sexo, tipo de esporte e experiência competitiva) sobre as variáveis critério (variáveis psicológicas do estudo), nos contextos de treinamento e de competição.

\section{Método}

O estudo é tipo descritivo, relacional e ex post facto. Importa destacar que é um estudo transversal impossibilitando a inferência causal.

\section{Participantes}

A amostra do presente estudo foi constituída de 357 atletas amadores, com idades compreendidas antre 18 e 40 anos $(M=25,69$, DP = 5,61), sendo 278 do sexo masculino e 79 do sexo feminino. Os atletas tinham entre 1 e 28 anos de experiência competitiva $(M=7,50$, DP = 5,77). Quando analisada a modalidade esportiva praticada, obteve-se a seguinte distribuição: futebol de campo $(n=89)$, futebol de salão $(n=92)$, voleibol $(n=56)$, handebol $(n=26)$, basquetebol $(n=22)$, esportes de combate $(n=47)$, surfe $(n=1)$, ténis $(n=1)$, triatlo $(n=1)$, natação $(n=9)$, corrida $(n=2)$, motocross $(n=10)$ e hipismo $(n=1)$. De modo geral, 72 atletas praticavam modalidades esportivas individuais, enquanto 285 atletas praticavam modalidades esportivas coletivas. Relativamente ao nível competitivo, 75 atletas afirmaram participar em competições de nível municipal/estadual, enquanto os restantes 282 atletas participavam em competições de nível regional/nacional. 


\section{Instrumento}

Para avaliar as habilidades psicológicas foi utilizado a versão brasileira (Fernandes et al., 2013) do Test of Performance Strategies-2(Hardy et al., 2010). O teste é constituído de 39 itens distribuídos em duas escalas que avaliam as habilidades psicológicas em situação de treino ou competição. A subescala de habilidades psicológicas na competição é composta por sete fatores: definição de objetivos (itens: 8 , 9, e 14), controle emocional (21, 22 e 29), automatização (24, 34 e 38), relaxamento (25, 32 e 35), imagética (11, 18 e 20), pensamentos negativos (4, 6 e 19) e ativação (28, 31 e 36). A subescala de habilidades psicológicas no treino é composta de seis fatores: definição de objetivos (1, 12 e 17), diálogo interno (2, 15 e 16), relaxamento (3, 7 e 30), imagética (5,13 e 23), automatização (10, 27 e 37) e ativação $(26,33,39)$. As afirmações foram respondidas de acordo com uma escala do tipo Likert de cinco pontos que tem como objetivo medir a intensidade destes constructos $(1=$ nunca a $5=$ sempre $)$. Os escores de cada fator são somados e divididos por três (número itens por fator), resultando em um escore por fator que pode variar de 1 a 5 .

\section{Procedimentos}

Previamente foram identificados os possíveis centros de trinamento de esportes na região nordeste do Brasil, especificamente, nas cidades de Ilhéus e Itabuna. Após a identificação, foi feito contato com esses centros de treinamento, através dos técnicos dos atletas, e obtidas as autorizações para a coleta de dados.

Após a devida autorização dos organizadores das competições e dos técnicos para a coleta de dados, os atletas foram informados dos objetivos da investigação e assinaram um termo de consentimento livre e esclarecido (TCLE), sendo garantidos o anonimato e a confidencialidade de todos os dados recolhidos. O preenchimento do questionário decorreu em ambiente calmo e sereno, de forma isolada ou em pequenos grupos de 5-10 atletas, e ocorreu aproximadamente uma hora antes do início das competições.

O presente estudo foi aprovado pelo Comitê de Ética em Pesquisa (Protocolo 425/2010) da Universidade Estadual de Santa Cruz (UESC).

\section{Análise Estatística}

A análise dos dados foi dividida em cinco estágios. Primeiramente, foram analisadas as premissas de normalidade, linearilidade, multicolinearilidade e homogeniedade da matriz de variância-covariância utlilizando frequência, scatter plots e Box's $M$ test. Segundo, examinamos a confiabilidade das dimensões da versão brasileira do TOPS-2 através do cálculo do alpha de Cronbach. No terceiro estágio, verificamos os coeficientes de correlação entre as dimensões do TOP-2. No quarto estágio foi empregado o método de split-median posto hoc para dicotomizar grupos em "alta" e "baixa" experiência competitiva baseado em a quanto tempo (em anos) o atleta participa de competições. Esta metodologia foi previamente utilizada por Fernandes, Nunes, Vasconcelos-Raposo e Fernandes (2014) que investigaram o efeito da experiência. Na sequência, no quinto estágio, separadamente os procedimentos de análise multivariada de variância (twoway MANOVA) para examinar o efeito isolado do sexo, tipo de esporte e experiência competitiva, assim como, o efeito combinado ou interativo dessas variáveis nas dimensões do TOPS-2, com pontos de corte de 0,20, 0,40, 0,80 representando efeitos de tamanhos pequenos, médios e grandes, respectivamente (Cohen, 1988). Para verificar o efeito do contexto (treino/competição) foi efetuado uma MANOVA para medidas repetidas. Por fim, no último estágio, foram realizadas análises de regressão múltipla para investigar o valor 
preditivo das variáveis explicativas sobre as variáveis critério, em ambos os contextos, treino e competição. Estas análises foram efetuadas no SPSS 22.0, sendo mantido o nível de significância em $5 \%(p<0,05)$.

\section{Resultados}

\section{Análise preliminar dos dados}

Os dados relativos aos escores das dimensões do TOPS-2 foram examinados com relação a possíveis erros de digitação, casos omissos e as premissas para a análise multivariada. Não foram registrados os casos omissos e os erros na entrada dos dados foram corrigidos, assim como, não houve casos extremos na análise multivariada e univariada.

As premissas de normalidade, linearilidade e multicolinearilidade não foram violadas, bem como a de homogeniedade da matriz de variância-covariância $(p>0,05)$ de acordo com as recomendações de Tabachnick e Fidell (2001). A consistência interna das dimensões de respostas doTOPS-2 foi calculada através do alpha de Cronbach e os índices revelados estavam acima do critério $\alpha$ \# 0.60 (Hair, Black, Babin, \& Anderson, 2014).

\section{Análise do efeito do sexo}

Os resultados das analises descritivas e da MANOVA por sexo (masculino vs feminino) são apresentados na Tabela 1. De modo geral, verificou-se que essa variável exerceu efeito isolado significativo $[F(13,342)=$ $2,131, p<0,05$; Wilks' Lambda $\left.=0,925, \eta p^{2}=0,075\right]$ sobre as habilidades psicológicas. Com base no valor do eta parcial quadrado $\left(\eta \mathrm{p}^{2}\right)$, o tamanho do efeito foi médio (Cohen, 1988). Além disso, a análise univariada subsequente permitiu verificar que o sexo exerceu efeito isolado significativo no relaxamento para a situação de treino $\left[F(1,355)=8,661, p<0,05 \eta p^{2}=0,024\right]$, no controle emocional para a situação de competição $[F$ $\left.(1,355)=7,004, p<0,01 ; \eta p^{2}=0,019\right]$ e no relaxamento para a situação de competição $[F(1,355)=13,326$, $\left.p<0,01 ; \eta p^{2}=0,035\right]$. Os tamanhos do efeito para essas análises, observando o eta parcial quadrado $\left(\eta p^{2}\right)$, foram pequenos.

Na sequência da análise univarida, foi verificado efeito significativo combinado ou interativo entre o sexo e o tipo de esporte na dimensão diálogo interno, na situação de treino $\left[F(1,353)=4,060, p<0,05 ; \eta p^{2}=0,011\right]$. 
TABELA 1

Análise comparativa dos fatores da versão brasileira do TOPS2 em função do sexo (feminino vs masculino)

\begin{tabular}{|c|c|c|c|c|}
\hline & $\begin{array}{l}\text { Feminino } \\
M \pm D P\end{array}$ & $\begin{array}{l}\text { Masculino } \\
M \pm D P\end{array}$ & $F$ & $\eta_{p}^{2}$ \\
\hline \multicolumn{5}{|l|}{ Treino } \\
\hline Definição de objetivos & $3,61 \pm 0.67$ & $3,54 \pm 0.82$ & 0,477 & 0,001 \\
\hline Automatização & $3,18 \pm 0.76$ & $3,25 \pm 0.79$ & 0,516 & 0,001 \\
\hline Relaxamento & $3,18 \pm 0,91$ & $2,81 \pm 1.00$ & $8,861^{\circ}$ & 0,024 \\
\hline Diálogo intemo & $3,85 \pm 0.69$ & $3,64 \pm 0.95$ & 3,299 & 0,009 \\
\hline Imagética & $3,24 \pm 0.62$ & $3,15 \pm 0.83$ & 0,745 & 0,002 \\
\hline Ativação & $3,34 \pm 0.63$ & $3,44 \pm 0.76$ & 1,282 & 0,004 \\
\hline \multicolumn{5}{|l|}{ Competição } \\
\hline Definição de objetivos & $3,90 \pm 0.82$ & $3,72 \pm 0.91$ & 2,439 & 0,007 \\
\hline Controle emocional & $2,20 \pm 0.95$ & $2,58 \pm 1.13$ & $7,004^{*}$ & 0,019 \\
\hline Automatização & $3,05 \pm 0.78$ & $3,17 \pm 0.82$ & 1,413 & 0,004 \\
\hline Relaxamento & $3,34 \pm 0.93$ & $2,87 \pm 1.02$ & $13,066^{* *}$ & 0,035 \\
\hline Imagética & $3,45 \pm 0.69$ & $3,37 \pm 0.91$ & 0,614 & 0,002 \\
\hline Ativação & $3,69 \pm 0.71$ & $3,61 \pm 0.80$ & 0,616 & 0,000 \\
\hline Pensamentos negativos & $2,15 \pm 0.80$ & $2,14 \pm 0.81$ & 0,000 & 0,616 \\
\hline
\end{tabular}

$$
\begin{aligned}
&{ }^{*} \mathrm{p}<0,05 \\
&{ }^{* *} \mathrm{p}<0,01
\end{aligned}
$$

\section{Análise do efeito do tipo de esporte}

Os resultados das análises descritivas e da MANOVA por tipo de esporte (individual vs coletivo) são apresentados na Tabela 2. De modo geral, verificou-se que essa variável não exerceu efeito significativo $[F$ $(13,343)=1,565, p \# 0,05$; Wilks' Lambda $\left.=0,944, \eta p^{2}=0,056\right]$ sobre as habilidades psicológicas. Com base no valor do eta parcial quadrado $\left(\eta \mathrm{p}^{2}\right)$, o tamanho do efeito foi médio. Além disso, a análise univariada subsequente permitiu verificar que o tipo de esporte somente exerceu efeito significativo sobre definição de objetivos, na situação de treino $\left[F(1,355)=10,124, p<0,01 \eta \mathrm{p}^{2}=0,028\right]$. O tamanho do efeito foi pequeno, com base no valor do eta parcial quadrado $\left(\eta \mathrm{p}^{2}\right)$.

$\mathrm{Na}$ sequência da análise univarida foi verificado efeito significativo interativo entre o tipo de esporte e a experiência competitiva nas dimensões: relaxamento na situação de treino $\left[F(1,333)=4,842, p<0,05 \eta p^{2}\right.$ $=0,014]$, relaxamento na situação de competição $\left[F(1,333)=5,270, p<0,05 \eta p^{2}=0,016\right]$ e ativação na 
situação de competição $\left[F(1,333)=4,601, p<0,05 \eta p^{2}=0,014\right]$. Com base no valor do eta parcial quadrado $\left(\eta \mathrm{p}^{2}\right)$, os tamanhos do efeito para essas análises foram todos pequenos.

TABELA 2

Análise comparativa dos fatores da versão brasileira do TOPS2 em função do tipo de esporte (individual vs coletivo)

\begin{tabular}{|l|l|l|l|l|}
\hline & Individual & Coletivo & $F$ & $\eta p^{2}$ \\
\hline Treino & $M \pm D P$ & $M \pm D P$ & \\
\hline Definição de objetivos & $3,81 \pm 0,76$ & $3,49 \pm 0,78$ & $10,124 *$ & 0,028 \\
\hline Automatização & $3,22 \pm 0,78$ & $3,24 \pm 0,78$ & 0,016 & 0,000 \\
\hline Relaxamento & $3,05 \pm 0,99$ & $2,85 \pm 0,99$ & 2,218 & 0,006 \\
\hline Diálogo intemo & $3,70 \pm 1,01$ & $3,68 \pm 1,01$ & 0,040 & 0,000 \\
\hline Imagética & $3,16 \pm 0,83$ & $3,17 \pm 0,77$ & 0,012 & 0,000 \\
\hline Controle atencional & $3,55 \pm 0,67$ & $3,39 \pm 0,75$ & 2,642 & 0,007 \\
\hline Competição & & & & \\
\hline Definição de objetivos & $3,83 \pm 0,94$ & $3,75 \pm 0,88$ & 0,469 & 0,001 \\
\hline Controle emocional & $2,49 \pm 1,12$ & $2,49 \pm 1,10$ & 0,000 & 0,000 \\
\hline Automatização & $3,13 \pm 0,93$ & $3,15 \pm 0,78$ & 0,039 & 0,000 \\
\hline Relaxamento & $3,07 \pm 1,04$ & $2,95 \pm 1,03$ & 0,767 & 0,002 \\
\hline Imagética & $3,49 \pm 0,89$ & $3,36 \pm 0,86$ & 1,339 & 0,004 \\
\hline Ativação & $3,68 \pm 0,72$ & $3,62 \pm 0,80$ & 0,133 & 0,000 \\
\hline Pensamentos negativos & $2,11 \pm 0,81$ & $2,15 \pm 0,80$ & 0,133 & 0,000 \\
\hline
\end{tabular}

\section{Análise do efeito da experiência competitiva}

Os resultados das análises descritivas e da MANOVA por experiência competitiva (baixa vs alta) são apresentados na Tabela 3. De modo geral, verificou-se que esta variável exerceu efeito isolado significativo $\left[F(13,323)=2,088, p \# 0,05\right.$; Wilks' Lambda $\left.=0,922, \eta \mathrm{p}^{2}=0,078\right]$ sobre as habilidades psicológicas, com um tamanho de efeito médio, dado o valor do eta parcial quadrado $\left(\eta \mathrm{p}^{2}\right)$. A análise univariada subsequente permitiu verificar que a experiência competitiva exerceu efeito significativo isolado sobre definição de objetivos na situação de treino $\left[F(1,355)=1,355, p<0,01 \eta p^{2}=0,053\right]$, sendo o tamanho do efeito também moderado, de acordo o valor do eta parcial quadrado $\left(\eta \mathrm{p}^{2}\right)$. 
$\mathrm{Na}$ sequência da análise univarida foi verificado efeito significativo e combinado entre experiência competitiva e sexo na definição de objetivos, na situação de treino $\left[F(1,333)=5,489, p<0,05 \eta p^{2}=0,016\right]$, ainda que o tamanho do efeito tenha sido pequeno.

TABELA 3

Análise comparativa dos fatores da versão brasileira do TOPS2 em função do experiência competitiva (baixa $v s$ alta)

\begin{tabular}{|l|l|l|l|l|}
\hline & Baixa & Alta & $F$ & $\eta p^{2}$ \\
& $M \pm D P$ & $M \pm D P$ & \\
\hline Treino & & & & \\
\hline Definição de objetivos & $3,50 \pm 0,77$ & $3,63 \pm 0,80$ & 2,331 & 0,007 \\
\hline Automatização & $3,19 \pm 0,81$ & $3,26 \pm 0,75$ & 0,882 & 0,003 \\
\hline Relaxamento & $2,95 \pm 1,02$ & $2,87 \pm 0,95$ & 0,510 & 0,002 \\
\hline Diálogo intemo & $3,65 \pm 0,93$ & $3,72 \pm 0,90$ & 0,408 & 0,001 \\
\hline Imagética & $3,15 \pm 0,78$ & $3,18 \pm 0,79$ & 0,090 & 0,000 \\
\hline Controle atencional & $3,46 \pm 0,70$ & $3,41 \pm 0,75$ & 0,310 & 0,001 \\
\hline Competição & & & & \\
\hline Definição de objetivos & $3,56 \pm 0,89$ & $3,97 \pm 0,85$ & $18,694 * *$ & 0,053 \\
\hline Controle emocional & $2,53 \pm 1,08$ & $2,48 \pm 1,13$ & 0,174 & 0,001 \\
\hline Automatização & $3,14 \pm 0,87$ & $3,14 \pm 0,75$ & 0,002 & 0,000 \\
\hline Relaxamento & $3,02 \pm 1,06$ & $2,95 \pm 0.98$ & 0,367 & 0,001 \\
\hline Imagética & $3,35 \pm 0,83$ & $3,41 \pm 0.90$ & 0,427 & 0,001 \\
\hline Ativação & $3,64 \pm 0,76$ & $3,67 \pm 0,81$ & 0,118 & 0,000 \\
\hline Pensamentos negativos & $2,13 \pm 0,81$ & $2,16 \pm 0,82$ & 0,083 & 0,000 \\
\hline & & & & \\
\hline & & & & \\
\hline & & & & \\
\hline
\end{tabular}

\section{Análise do efeito do contexto (treino/competição)}

Os resultados das analises descritivas e da MANOVA por contexto são apresentados na Tabela 4. De modo geral, verificou-se que esta variável exerceu efeito significativo $[F(5,352)=17,028, p \# 0,01$; Wilks' Lambda $\left.=0,805, \eta p^{2}=0,195\right]$ sobre as habilidades psicológicas, e o tamanho do efeito de acordo com o valor do eta parcial $\left(\eta \mathrm{p}^{2}\right)$ foi grande. A análise univariada subsequente permitiu verificar que o contexto exerceu efeito isolado significativo sobre definição de objetivos $\left[F(1,356)=28,127, p<0,01 \eta p^{2}=0,073\right]$, automaticidade $\left[F(1,356)=5,689, p<0,05, \eta \mathrm{p}^{2}=0,016\right]$, relaxamento $\left[F(1,356)=4,641, p<0,05, \eta \mathrm{p}^{2}=\right.$ $0,013]$ e imagética $\left[F(1,356)=29,864, p<0,01, \eta p^{2}=0,017\right]$. O tamanho do efeito para definição de objetivos foi médio e para as demais variáveis foi pequeno. 
TABELA 4

Análise comparativa dos fatores da versão brasileira do TOPS2 em função do contexto (treino $v$ s competição)

\begin{tabular}{|l|l|l|l|l|}
\hline & Situação de & Situação de & & \\
& Treino & Competição & $F$ & $\eta_{p^{2}}$ \\
& $M \pm D P$ & $M \pm D P$ & & \\
\hline Definição de objetivos & $3,55 \pm 0,79$ & $3,76 \pm 0,89$ & $28,127 * *$ & 0,073 \\
\hline Automatização & $3,23 \pm 0,78$ & $3,15 \pm 0,81$ & $5,689 *$ & 0,016 \\
\hline $\begin{array}{l}\text { Relaxamento } \\
\text { Imagética }\end{array}$ & $2,89 \pm 0,99$ & $2,97 \pm 1,01$ & $4,641^{*}$ & 0,013 \\
\hline
\end{tabular}

$$
\begin{aligned}
{ }^{*} \mathrm{p} & <0,05 \\
{ }^{*} \mathrm{p} & <0,01
\end{aligned}
$$

\section{Análises de Regresssão Múltipla}

Adicionalmente, foram realizadas análises de regressão múltipla (Stepwise) para investigar o valor preditivo das variáveis explicativas (sexo, tipo de esporte e experiência competitiva) sobre as variáveis psicológicas em ambos os contextos: de treinamento e de competição. Os resultados dessas análises estão apresentados na Tabela 5 e 6.

TABELA 5

Modelos de Regressão hierárquica (Stepwise Method)

\begin{tabular}{|c|c|c|c|c|c|c|c|}
\hline & & \multicolumn{3}{|c|}{ Definição de objetivos } & \multicolumn{3}{|c|}{ Relaxamento } \\
\hline Step & $\begin{array}{l}\text { Preditores do } \\
\text { Modelo }\end{array}$ & $\beta$ & $\mathrm{t}$ & $\mathrm{p}$ & $\beta$ & $\mathrm{t}$ & $\mathrm{p}$ \\
\hline 1 & Sexo & 0,48 & 0,90 & 0,37 & 0,14 & 2,53 & 0,012 \\
\hline 2 & Tipo de esporte & -21 & $-3,69$ & 0,000 & $-0,89$ & $-1,65$ & 0,099 \\
\hline 3 & $\begin{array}{l}\text { Expenencia } \\
\text { competitiva }\end{array}$ & 0,14 & 2,53 & 0,012 & 0,032 & $-0,60$ & 0,552 \\
\hline $\begin{array}{l}\text { Modelo } \\
\text { estatístico }\end{array}$ & & & & & & & \\
\hline $\mathrm{F}(2,334)$ & & $8,02^{* *}$ & & & $\mathrm{~F}(1,335)$ & $6,41^{*}$ & \\
\hline $\mathrm{R}^{2}$ & & 0,046 & & & & 0,019 & \\
\hline
\end{tabular}
para as variáveis psicológicas no contexto de treinamento

Nota Valores de F e $\mathrm{R}^{2}$ para Modelos Finais de Regressão

$$
\begin{aligned}
& { }^{* *} \mathrm{p}<0,01 \\
& { }^{*} \mathrm{p}<0,05
\end{aligned}
$$


Como é possível observar na Tabela 5, no que concerne à variável definição de objetivos, tipo de esporte e experiência competitiva, foram as melhores preditoras, Nesse modelo, o coeficiente de correlação ao quadrado é fraco $\left(\mathrm{r}^{2}=0,05\right)$, o que significa que $5 \%$ da variância em definição de objetivos pôde ser explicada pelo de tipo de esporte e experiência competitiva dos atletas. Por outro lado, apenas o sexo dos atletas predisse relaxamento, sendo que apenas $2 \%$ da variância da variável relaxamento pôde ser explicada pelo sexo dos atletas $\left(r^{2}=0,02\right)$.

TABELA 6

Modelos de Regressão hierárquica (Stepwise Method) para as variáveis psicológicas no contexto de competição

\begin{tabular}{|c|c|c|c|c|c|c|c|c|c|c|}
\hline & & Definiçãc & de o & etivos & & role emo & onal & & laxam & \\
\hline Step & $\begin{array}{l}\text { Preditores } \\
\text { do Modelo }\end{array}$ & $\beta$ & $\mathrm{t}$ & $\mathrm{p}$ & $\beta$ & $\mathrm{t}$ & $\mathrm{p}$ & $\beta$ & $\mathrm{t}$ & $\mathrm{p}$ \\
\hline 1 & Sexo & 0,07 & 1,39 & 0,165 & $-0,16$ & $-3,02$ & 0,003 & 0,16 & 2,99 & 0,003 \\
\hline 2 & $\begin{array}{l}\text { Tipo de } \\
\text { esporte }\end{array}$ & $-0,10$ & $-1,90$ & 0,058 & 0,01 & 0,11 & 0,91 & $-0,06$ & $-1,10$ & 0,272 \\
\hline 3 & $\begin{array}{l}\text { Experiência } \\
\text { competitiva }\end{array}$ & 0,23 & 4,32 & 0,000 & $-0,03$ & $-0,57$ & 0,56 & $-0,02$ & $-0,47$ & 0,642 \\
\hline & $\begin{array}{l}\text { Modelo } \\
\text { estatístico }\end{array}$ & & & & & & & & & \\
\hline & $\mathrm{F}(1,335)$ & $18,69^{*}$ & & & $335)$ & $9,13 * *$ & & & & \\
\hline & $\mathrm{R}^{2}$ & 0,053 & & & & 0,027 & & & 0,026 & \\
\hline
\end{tabular}

Nota Valores de F e $\mathrm{R}^{2}$ para Modelos Finais de Regressão

$$
\begin{aligned}
& { }^{* *} \mathrm{p}<0,01 \\
& { }^{*} \mathrm{p}<0,05
\end{aligned}
$$

A Tabela 6 mostra que experiência competitiva predisse a definição de objetivos dos atletas. No modelo final de regressão, o coeficiente de correlação ao quadrado é fraco $\left(r^{2}=0,05\right)$, o que significa que $5 \%$ da variância em definição de objetivos pôde ser explicada pela experiência competitiva. No que concerne ao controle emocional, apenas a variável explicativa sexo foi preditora, sendo que apenas 3\% da variância nos níveis de controle emocional pôde ser explicada pelo sexo dos atletas $\left(\mathrm{r}^{2}=0,03\right)$. Finalmente, sexo também predisse relaxamento, sendo responsável por explicar uma variância de em torno de $3 \%$ dos níveis dessa variável psicológica. 


\section{Discussão}

O presente estudo teve como obejtivos: i) verificar a correlação entre as habilidades psicológicas, ii) examinar os efeitos do contexto (treino vs competição), experiência competitiva (baixa $v s$ alta), tipo de esporte (individual $v s$ coletivo) e do sexo (masculino $v$ feminino) no uso das habilidades psicológicas e iii) investigar o poder preditivo das variáveis explicativas (sexo, tipo de esporte e experiência competitiva) sobre as variáveis critério (variáveis psicológicas do estudo), nos contextos de treinamento e de competição. É o primeiro estudo no Brasil com a versão brasileira do TOPS-2, proporcionado uma compressão do efeito isolado e combinado de variáveis (sexo, tipo de esportes e experiência competitiva) que podem interferir nas habilidades psicológicas de atletas.

O sexo demonstrou efeito significativo na análise multivariada e na univariada para as dimensões relaxamento e controle emocional, na situação de treino, e para a dimensão relaxamento, na situação de competição. Sexo também foi o único preditor de relaxamento, na situação de treino, e de controle emocional e de relaxamento, na situação de competição, ainda que seu poder explicativo tenha sido pequeno nos modelos finais regressão, o que é normalmente esperado em estudos que envolvem variáveis psicológicas complexas. Esses resultados estão de acordo com reportados por Fernandes et al., (2013), e Fernandes e Fernandes (2015), principalmente com relação à variável relaxamento, que concluíram que atletas masculinos e femininos apresentam níveis de relaxamento significativamente diferentes, tanto nas situações de treino como nas de competição. Mais especificamente, as atletas reportaram sentirem-se mais relaxadas do que os atletas, que por sua vez, por não estarem relaxados o suficiente, exibiram níveis menores de controle emocional, se comparadas às atletas. Da mesma forma, o estudo de Hagan et al. (2017) e Kristjansdottir et al. (2018), corrobora parcialmente com os dados do presente estudo, na medida em que os autores encontraram diferenças significativas na utilização de habilidades psicológicas entre homens e mulheres, em especial no uso do diálogo interno. Com relação ao efeito combinado do sexo e do tipo de esporte, essa interação foi significativa para diálogo interno na situação de treino, ou seja, os atletas masculinos e femininos, quer seja para esportes individuais ou para esportes coletivos, utilizam de forma significativamente diferente esse tipo de habilidade psicológica.

O tipo de esporte (individual/coletivo) e a experiência competitiva tiveram efeitos sobre a dimensão definição de objetivos, e quando testados os modelos de regressão ambos foram bons preditores de definição de objetivos, na situação de treino, enquanto que na situação de competição, apenas experiência competitiva predisse definição de objetivos. Especificamente, os escores de definição de objetivos foram em média significativamente mais altos para os esportes individuais, na situação de treino, mas não na situação de competição corroborando com os achados de Fernandes et al. (2013). Para Weinberg, Stitcher e Richardson (1994), a definição de objetivos consiste numa importante fonte de automotivação, sendo o seu desenvolvimento fundamental para os esportes individuais onde a componente equipe/time não está presente para auxiliar na motivação de atletas. É importante observar que na situação de competição, os escores relativos à definição de objetivos foram mais altos do que na situação de treino. Isso permite deduzir que essa dimensão associada a automotivação está mais presente em situações reais de competição, nas quais os atletas precisam estabelecer objetivos de desempenho, objetivos específicos de resultado, e avaliar o quanto eles atingem os objetivos da competição. Com relação à experiência competitiva, nota-se que os atletas com menos experiência competitiva tiveram menores escores de definição de objetivos comparados aos atletas com mais experiência, ou seja, os menos experientes não estavam utilizando essa habilidade, como os mais experientes, para aumentar a sua motivação, resultado que corrobora com os achados de Weinberg, Stitcher e Richardson (1994). 
No que diz respeito às análises interativas, as variáveis sexo e experiência competitiva exerceram um efeito combinado sobre a definição de objetivos dos atletas. Ou seja, atletas femininas com mais experiência competitiva reportaram maiores escores de definição de objetivos. Dessa forma, é sugerido treino especializado e personalizado para esses subgrupos com o objetivo de desenvolver a habilidade psicológica definição de objetivos, especifico para atletas masculinos e femininos, assim como, para esportes individuais e coletivos, e atletas com alta e baixa experiência competitiva.

Da mesma forma, na análise interativa entre tipo de esporte e experiência competitiva o efeito foi significativo para relaxamento, tanto no treino, como na competição, e para a variável ativação, em situação de competição. Atletas de esportes coletivos e com maior experiência competitiva, tanto em situação de treino como de competição, tendem a apresentar menores escores de relaxamento, provavelmente porque essas situações são mais ansiogênicas ou tensas, dificultando a habilidade de relaxar. Sabe-se, por exemplo, que a emoção é contagiante, e em situações de grupo o nervosismo de uns pode desencadear o nervosismo dos demais, principalmente naquelas situações em que o atleta já acumula mais experiências negativas de derrotas, que é o caso dos atletas mais experientes. Em virtude de o relaxamento ser utilizado de forma diferente para tipo de esporte (individual/coletivo) e experiência competitiva (alta/baixa), entende-se que são necessárias formas de intervenções específicas para cada grupo com o objetivo de desenvolver essa habilidade. Horn et al. (2011) confiram em seus estudos que os atletas são beneficiados com programas de intervenção para desenvolvimento de habilidades psicológicas como o relaxamento. No que diz respeito à ativação, atletas com mais experiência competitiva e de esportes individuais parecem ter mais capacidade de se concentrar melhor para alcançar o nível de energia requerido para desempenhar bem numa competição.

Finalmente, foram realizadas análises para investigar o efeito do contexto esportivo (treino ou competição) sobre o uso das habilidades psicológicas avaliadas pela versão brasileira do TOPS-2. Como era de ser esperar, nas situações de competição foram observados os maiores escores de algumas habilidades psicológicas, como definição de objetivos, relaxamento e imagética. Se pensarmos que as situações de competição são mais tensas ou ansiogênicas do que as situações de treino, conseguimos entender porque nessas situações se faz mais necessário desenvolver a capacidade de estabelecer objetivos específicos, relacionados a resultados específicos que se pretende alcançar e de se avaliar se esses objetivos foram plenamente atingidos. Seguindo o mesmo raciocínio, entende-se que desenvolver a capacidade de relaxar frente a pressões exercida por situações tensas e desenvolver técnicas imagéticas que permitem vivenciar ou imaginar como será o desempenho e como a competição irá transcorrer tendem a ser mais requeridas em situações de competição do que de treino. Por outro lado, a habilidade psicológica automatização foi mais utilizada nos treinos do que na competição. A dimensão automatização diz respeito à capacidade de desempenhar uma tarefa, nesse caso a esportiva, sem pensar muito, ou seja, fazê-lo na forma instintiva ou de piloto automático. É razoável pensar que sob a pressão da situação competitiva os atletas tenham sentido maior dificuldade de automatizar seus movimentos técnicos.

O presente estudo encontrou efeitos isolados e interativos das variáveis sexo/tipo de esporte, tipo de esporte/experiência competitiva, sexo/experiência competitiva sobre algumas habilidades psicológicas estudadas, em especial sobre definição de objetivos, dialogo interno e relaxamento. Vealey (2007) e Williams e Harris (2001), indicam que o treinamento dessas habilidades psicológicas é fundamental para melhorar o desempenho dos atletas no âmbito da Psicologia do Esporte. Dessa forma, é sugerido que Psicólogos dos Esportes tenham um olhar atento para essas habilidades com o objetivo de desenvolvê-las com intervenções específicas, levando em consideração as diferenças de grupo, com o objetivo de maximizar o desempenho dos atletas. 
O presente estudo tem algumas limitações, a saber: i) a amostra foi do tipo intencional e não probabilística e tais tipos de amostra podem não ser representativas da população de atletas de competição; ii) esse é primeiro estudo publicado no contexto esportivo brasileiro com a versão brasileira do TOPS-2, portanto seus resultados são ainda iniciais, sendo de fundamental importância que estudos futuros continuem o processo de investigação e confirmação dos resultados obtidos; iii) a amostra não estava em número equivalente entre sexo e tipos de esportes; iiii) a amostra foi constituída de atletas amadores; iiii) o estudo foi do tipo transversal impossibilitando inferências causais. É necessário que pesquisadores brasileiros avancem para estudos longitudinais e intervencionistas com relação às habilidades psicológicas, a exemplo do estudo de Hagan et al. (2017), assim como realizem estudos que utilizem amostras de atletas de elite. Para Freitas, Dias e Fonseca (2013) as pesquisas sobre habilidades psicológicas tiveram um considerável crescimento nos últimos anos, no entanto, ainda há muito a ser explorado e futuras pesquisas são necessárias.

A Psicologia do esporte tem se preocupado, além de estudos descritivos e correlacionais, com estudos experimentais que verificam o efeito de programas de treinamento de habilidades psicológicas específicas para alguns esportes, como por exemplo, ginastas (Woodman, Zourbanos, Hardy, Beattie, \& Mcquillan, 2010), esgrima (Abdel-Rahman, \& Magdy, 2014) e taekwondo (Lim, \& O 'Sulivan, 2016), tendo na suas conclusões que as habilidades psicológicas podem ser melhoradas com programas de treinamento, objetivando um melhor desempenho. Dessa forma, programas de desenvolvimento de habilidades psicológicas são de fundamental importância para os esportes competitivos e o presente estudo sugere de que esses programas podem ser específicos levando em consideração o sexo, esportes individuais e coletivos, e a experiência competitiva dos atletas. Para Sadeghi et al. (2010), psicólogos do esporte devem prestar atenção às diferenças individuais, assim como, técnicas específicas e necessidades psicológicas de um determinado esporte. Além disso, explicitando a importância de se considerar os aspectos psicológicos envolvidos no esporte, afirmam que o conhecimento de certas características de um esporte e o entendimento profundo dos pensamentos e comportamentos dos atletas antes, durante e depois da competição auxiliam os atletas a encontrarem o seu melhor desempenho.

\section{ConClusão}

O sexo, tipo de esportes e experiência competitiva tiveram efeito significativo no relaxamento, controle emocional e definição de objetivos. Além disso, o contexto (treino ou competição) teve efeito significativo sobre definição de objetivos, automatização, relaxamento e imagética. A interação entre sexo/tipo de esportes teve efeito sobre diálogo interno (treino), e tipo de esportes/experiência competitiva teve efeito sobre o relaxamento (treino e competição) e ativação (competição). Finalmente, experiência competitiva/sexo teve efeito significativo e combinado sobre definição de objetivo (treino). Assim, foi evidenciado, no presente estudo, o efeito de variáveis isoladas e combinadas sobre as habilidades psicológicas de atletas, e observaram-se especificidades de seu uso para os contextos de treino ou competição. Dessa foram, sugere-se que programas de treinamento de habilidades psicológicas levem em consideração especificidades de grupo, como sexo, tipo de esportes e experiência competitiva, bem como considere o contexto em que o atleta vai desempenhar sua atividade esportiva.

\section{REFERÊNCIAS}

Abdel-Rahman, O., \&, Magdy, S. (2014). The impact of green exercise on test of performance strategies, physical variable sand counter-time performance for Egyptian Epee fencers. Ovidius University Annals, Series Physical Education and Sport/Science, Movement and Health, 14(1), p.154-155. Disponível em: https://www.iat.uni-lei pzig.de/datenbanken/iks/sponet/Record/4031532 
Burton, D., \&, Raedeke, T. (2008) Sport psychology for coaches. Champaign, IL: Human Kinetics.

Craft, L. L., Magyar, T. M., Becker, B. J., \&, Feltz, D. L. (2003). The relations between the Competitive State Anxiety Inventory-2 and sport performance: A meta-analysis. Journal of Sport and Exercise Psychology, 25, 44-65. Disponível em: https://journals.humankinetics.com/doi:10.1123/jsep.25.1.44

Cohen, J. (1988). Statistical power analysis for the behavioral sciences. Hillsdale, New Jersey: Lawrence Erlbaum Associates.

Edwards, C., Tod, D., \& McGuigan, M. (2008). Self-talk influences vertical jump performance and kinematics in male rugby union players. Journal of Sports Sciences, 9(1), 1-7. Disponível em: https://www.ncbi.nlm.nih.gov/pubm ed/18923954/doi:10.1080/02640410802287071.

Fernandes, M. G., Vasconcelos-Raposo, J., \& Fernandes, H. M. (2013). Propriedades psicométricas da versão brasileira do TOPS2. Revista Ibero-Americana de Diagnóstico e Avaliação Psicológica, 36(2), 71-98. Disponível em: https ://dialnet.unirioja.es/servlet/articulo?codigo $=5064337$

Fernandes, M. G., Vasconcelos-Raposo J., \& Fernandes H. M. (2012). Propriedades psicométricas do CSAI-2 em atletas brasileiros. Psicologia: Reflexão e Crítica, 25(4), 679-687. Disponível em: http://www.scielo.br/scielo.ph p?script=sci_arttext\&pid=S0102-79722012000400007/doi:10.1590/S0102-79722012000400007

Fernandes, M. G.; Nunes, S. A. N., Vasconcelos, R., J., Fernandes, H. M., \& Brustad, R. (2013). The CSAI-2: An examination of the instrument's factorial validity and reliability of the intensity, direction and frequency dimensions with Brazilian athletes. Journal of Applied Sport Psychology, 25(4),377-391. Disponível em: https:// www.tandfonline.com/doi:10.1080/10413200.2012.744780?journalCode=uasp20

Fernandes, M. G.; Nunes, S. A. N. ; Vasconcelos-Raposo, J., \& Fernandes, H. M. (2014). Efeitos da experiência nas dimensões de intensidade, direção e frequência da ansiedade e autoconfiança competitiva: Um estudo em atletas de desportos individuais e coletivos. Motricidade, 10(2), p. 81-89. Disponível em: http://www.scielo.mec.pt/sci elo.php?script=sci_arttext\&pid=S1646-107X2014000200009/doi:10.6063/motricidade

Fernandes, M. G., \& Fernandes, H. M. (2015). Tradução, adaptação transcultural e propriedades psicométricas da versão brasileira do Test of Performance Strategies. Psicologia Reflexão e Critica, 28(1), 136-146. Disponível em: http://www.scielo.br/scielo.php?script=sci_arttext\&pid=S0102-79722015000100136/doi:10.1590/167 8-7153.201528115.

Freitas, S., Dias, C., \& Fonseca, A. (2013). Psychological skills training applied to Soccer: A systematic review based on research methodologies. Review of European Studies, 5(5), 18-29. Disponível em: https://www.ccsenet.org/j ournal/index.php/res/article/view/29680/doi:10.5539/res.v5n5p18

Gould, D., Flett, M. R., \& Bean, E. (2009). Mental preparation for training and competition. In: Sport Psychology. Wiley-Blackwell., p. 53-63.

Gould, D., \& Weinberg, R. S. (2015). Foundations of sport and exercise psychology: Study guide. Champaign: Human Kinetics.

Hagan, J.E., Jnr., Pollmann, D., \& Schack, T. (2017). Exploring temporal patterning of Psychological skill susage during the week leading up to competition: Lessons for developing intervention programmes. PLoS ONE 12(8): e0181814. Disponível em: https://doi:10.1371/journal.pone.0181814.

Hair, J., Black, W., Babin B., \& Anderson, R. (2014). Multivariate data analysis. Harlow: Pearson Education Limited. 2014.

Hardy, J. (2006). Speaking clearly: A critical review of the self-talk literature. Psychologyof Sport and Exercise,7(1), 81-97. Disponível em: https://www.sciencedirect.com/science/article/pii/S1469029205000476

Hardy, L., Roberts, R., Thomas, P. R., \& Murphy, S. M. (2010). Test of Performance Strategies (TOPS): Instrument refinementusing confirmatory factor analysis. Psychology of Sport and Exercise, 11(1), 27-35. doi:10.1016/ j.psychsport.2009.04.007. Disponível em: https://www.sciencedirect.com/science/article/pii/S146902920900 0612/doi:10.1016/j.psychsport.2005.04.002 
Horn, C. M., Gilbert, J. N., Gilbert, W., \& Lewis, D. K. (2011). Psychological skills training with community college athletes: The UNIFORM approach. The Sport Psychologist, 25, 321-340. Disponível em: https://journals.hum ankinetics.com/doi:10.1123/tsp.25.3.321

Kristjansdottir, H., Erlingsdottir, A. V., Sveinsson, G. \& Saavedra, J. M. (2018). Psychological skills, mental toughness and anxiety in elite handball players. Personality and Individual Differences, 134, 125-130. Disponível em: http s://www.sciencedirect.com/science/article/pii/S0191886918303404

Kulmatycki, L. A., \& Bukowska, K. (2007). Differences in experiencing relaxation by sport coaches in relation to sport type and gender. Human Movement, 8(2), 98-103.

Lane, A. M., Harwood, C., Terry, P. C., \& Karageorghis, C. I. (2004). Confirmatory factor analysis of the Test of Performance Strategies (TOPS) among adolescent athletes.Journal of Sports Sciences, 22(9), 803-812. Disponível em: https://www.ncbi.nlm.nih.gov/pubmed/15513274/doi:10.1080/02640410410001716689

Lim, T \& O Sulivan, D, M. (2016). Case Study of Mental Skills Training for a Taekwondo Olympian. Journal Human Kinetics, 50, 235-245. Disponível em: https://www.ncbi.nlm.nih.gov/pmc/articles/PMC5260659/doi:10.15 15/hukin-2015-0161

Neil, R., Mellalieu, D.S., \& Hanton, S. (2006). Psychological skills usage and the competitive anxiety response as a function of skill level in rugby union. Journal of Sport Science and Medicine, 5, 415-423. Disponível em: https:/ /www.ncbi.nlm.nih.gov/pmc/articles/PMC3842142/

Newmark, T. S., \& Bogacki, D. F. (2005). The use of relaxation, hypnosis, and imagery in sport psychiatry. Clinics in Sports Medicine, 24(4), 973-977. Disponível em: https://www.ncbi.nlm.nih.gov/pubmed/16169457/doi:10.1 016/j.csm.2005.06.003

Sadeghi, H., Omar-Fauzee, M. S., Jamalis, M., Ab-Latiff, R., \& Cheric, M. (2010). The mental skills training of university soccer players. International Education Studies, 3, 81- 90. Disponível em: http://www.ccsenet.org/jo urnal/index.php/ies/article/view/4928

Tabachnick, B. G., \& Fidell, L. S. (2001). Using Mul-tivariate Statistics. New York: Harper \& Row.

Thomas, P. R.; Murphy S. M., \& Hardy L. (1999). Test of Performance Strategies: development and preliminary validation of a comprehensive measure of athletes' psychological skills. Journal of Sports Sciences, 17, 697-711. Disponível em: https://www.ncbi.nlm.nih.gov/pubmed/10521001/doi:10.1080/026404199365560

Vealey, S. R. (2007). Mental skills training in sport. In G. Tenenbaum, R. Eklund and R. Singer (Eds.), Handbook of sport psychology. New Jersey: Wiley.

Weinberg, R. S., Stitcher, T., \& Richardson P. (1994). Effects of a seasonal goal setting program on lacrosse performance. The Sport Psychologist, 8, 166-175. Disponível em: https://journals.humankinetics.com/doi:10.1 $123 /$ tsp.8.2.166

Williams, J. M., \& Harris, V. D. (2001). Relaxation and energization technique for regulation of arousal. In J. M. Williams (Ed.), Applied sport psychology: Personal growth to peak performance. California: Mayfield.

Wilson, D., Bennett, E. V., Mosewich, A. D., Faulkner, G. E., Crocker, P. R. E. (2019). "The zipper effect": Exploring the interrelationship of mental toughness and self-compassion among Canadian elite women athletes. Psychology of Sport and Exercise, 40, 61-70. Disponível em: https://www.sciencedirect.com/science/article/pii/S1469029 218301973

Woodman, T., Zourbanos, N., Hardy, L., Beattie S., \& Mcquillan, A. (2010). Do performance strategies moderate the relationship between personality and training behaviors? An exploratory study. Journal of Applied Sport Psychology, 22, 183-197. Disponível em: https://www.tandfonline.com/doi:10.1080/10413201003664673 